\title{
THE NEW SIMULTANEOUS PVDF/VISAR MEASUREMENT TECHNIQUE: APPLICATIONS TO HIGHLY POROUS HMX
}

\author{
M. U. Anderson and R. A. Graham
}

RECEIVED

Sandia National Laboratories, Albuquerque, NM 87185-1421

DEC 271995

$\mathrm{O} \mathrm{S} T \mathrm{I}$

Time-resolved pressure and particle velocity measurements provide the foundation for modern descriptions of rate-dependent materials deformation processes in condensed matter subjected to high pressure shock loading. Much of that foundation has been based on the VISAR, nanosecond time-resolved particle velocity technique. It has been known for sometime that full description of the rate-dependent processes requires both particle velocity and stress data, but stress sensors with nanosecond resolution have not been previously available for general usage. More recently, the piezoelectric polymer PVDF has been used for stress and stress-rate measurements in rate dependent materials response experiments. Neither method, by itself, gives unique material response models, particularly in the case of highly porous materials. The first simultaneous, time-resolved measurements with VISAR and PVDF have now been carried out. Measurements were taken on highly porous HMX that clearly show the two-dimensional nature of HMX viscous compression behavior, followed by the onset of chemical reaction. This technique promises to provide a qualitative improvement in our ability to develop rate-dependent material descriptions of all solids including highly porous solids. Precise use of this technique will require new window materials.

\section{INTRODUCTION}

A new technique has been developed to study materials under shock compression by simultaneous measurement of material response in both the stress and volume planes. These simultaneous measurements allow unique observations of timedependent material response under shock compression. Time-dependent material response occurs during shock compression of materials in the inert and reactive states, and is particularly apparent in the case of highly porous materials.

Highly porous materials are expected to have time-dependent behavior since a typical sample initially consists of particles in contact with other particles at a finite number of locations, while the rest of the surface area is in contact with voids. A shock wave propagating into this highly porous matrix causes localized stresses at particle contact points, resulting in large shear deformation, and local regions of zero stress from the unsupported particle faces. As the material begins to consolidate toward solid density, each particle is subject to deformation in the form of fracture, shear, and plastic flow. The portions of each particle that are unsupported complicate this deformation process due to the zero stress regions. Thus, many localized deformation fronts are in constant communication with localized voids, and the material deformation that results has material, morphology and densitydependent time scales over which mechanical equilibrium is achieved. Prior work with PVDF measurements has provided clear evidence for the rate-dependent behaviors of a number of powder samples. (1)

\section{EXPERIMENTAL TECHNIQUE}

The simultaneous PVDF/VISAR measurement technique developed in the present study was carried out on the powder samples subjected to controlled shock-loading from a compressed-gas gun. The sample is encased in a polymer capsule with PVDF gauge packages at the impact surface, and at sample

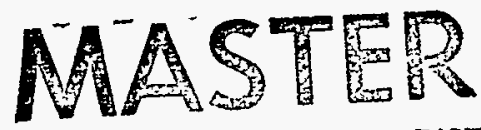


input and propagated locations as in Figure 1. The propagated-location PVDF gauge package is mounted directly on the VISAR reflector which is sputtered to a PMMA window.

The piezoelectric polymer PVDF is a $25 \mu \mathrm{m}$ thick film that generates a stress-rate dependent current. (2) The current is recorded electronically, then numerically integrated to give the piezoelectric charge-versus-time profile. (3) The shock response of PVDF is used to convert the charge-versus-time to stress-versus-time. The particle velocity measurements of the present study use the VISAR interferometer to measure mirror motion-versustime as evidenced by the Doppler shift of the laser light returned from the mirror through reference and delayed optical paths. Two separate interferometers having complementary delay constants were used in each experiment for enhanced accuracy.

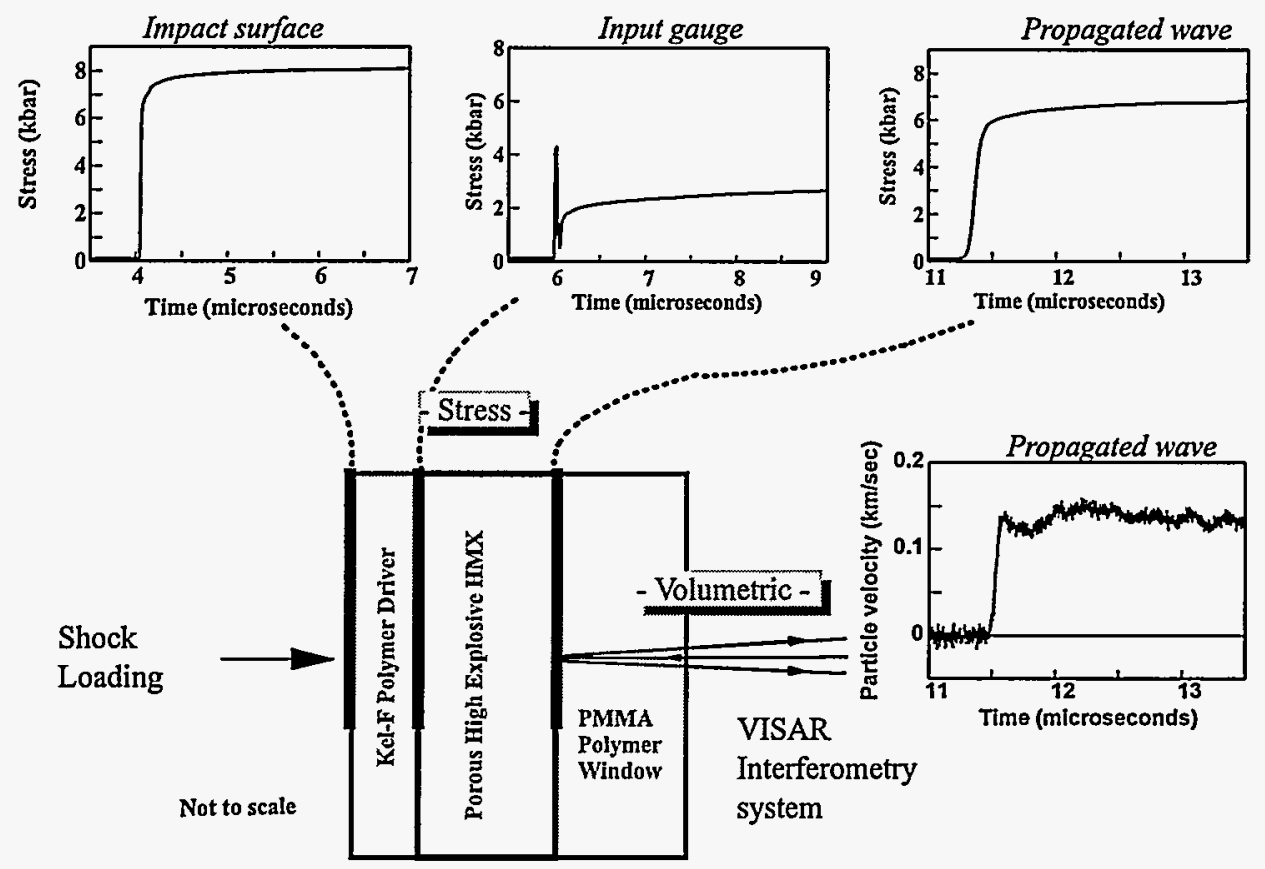

FIGURE 1. Simultaneous measurements in stress and volume planes are made with PVDF/VISAR technique. The experiment includes impact surface stress measurements with PVDF as well as PVDF measurements of time-resolved pressure of both the input and propagated stress pulses in the porous HMX high explosive sample. The propagated-location PVDF gauge package is mounted directly on the VISAR reflector which is sputtered to a PMMA window. Resulting stress-versus-particle velocity data gives unique material description of complex two-dimensional dynamic deformation of the highly porous materials

\section{RESULTS}

Figure 1 shows the three PVDF measurements and the VISAR measurements on the porous HMX sample. The $64 \%$ dense HMX sample as is described by Gustavsen. (4) The impact surface PVDF gauge provides a detailed record of the impact stress while the input gauge to the powder sample provides the initial stress signature input to the sample. This record clearly shows the 
importance of the viscoelastic behavior of the Kel F driver. The early time "spike" is a result of the gauge signal prior to the arrival of the wave reflected from the powder interface. The propagated wave PVDF record shows the arrival of a dispersed wave with a continuously rising stress history. The VISAR record for the propagated wave also shows a dispersed wave with relaxation.

The measurements of both stress or particle velocity-versus-time show time-dependent material response with significant rate dependence, but the records show that neither is particularly distinguished. The individual records show no explicit detailed features which can be connected to the deformation. Nevertheless, when the measurements are combined and plotted in the stress-versus-particle plane, as shown in Figure 2, distinctive features of the material response can be identified.

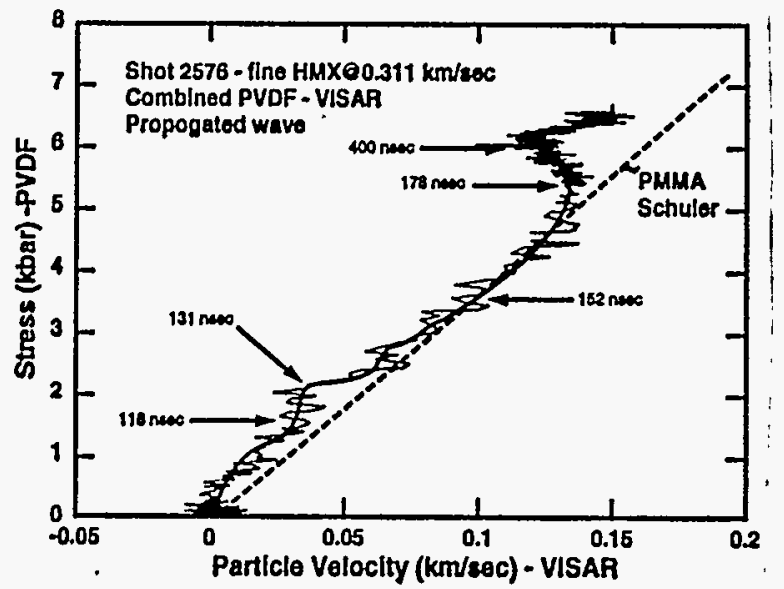

Figure 2. The time-dependent stress-versus-particle velocity relationship determined from simultaneous PVDF and VISAR measurements show detail on the deformation of the porous HMX sample as it deforms from the distended to the fully dense state. The data are shown by the discontinuous line which is fit by the solid line. The various times noted indicate the relationship at that particular time. The records indicate an approach to solid density at about 130 nsec followed by a quiescent period and an apparent chemical reaction.

The velocity-stress record shows a behavior for the first approximately 140 nanoseconds of response which lies above the relationship of the PMMA. Since the powder and PMMA are in contact, they must have equal states. States off the PMMA one dimensional behavior are clear indications of the two-dimensional deformation of the powder sample. Such behavior is expected for the heterogeneous, multidimensional deformation. After the early period the response moves to the PMMA response relationship for one-dimensional conditions. This observation indicates that the powder is now fully compacted to densities approaching solid density. After a delay time, the upward excursion of the relationship is consistent with the onset of limited chemical reaction. Thus, the measurements appear to provide a detailed picture of the deformation process from the initial state to solid density.

Mechanical impedance mismatches between the PVDF gauge package and the PMMA cause a small effect of wave reverberation for the first 20 nanoseconds. This difficulty can be overcome with the use of amorphous Kel F windows. Experiments to describe the index of refraction for the windows are in progress.

\section{CONCLUSIONS}

The present work has shown the first simultaneous measurements of particle velocity and stress with time resolutions of a few nanoseconds.

The technique should prove generally useful for observations on a wide variety of solids in the solid state and in porous states. Most existing descriptions of rate-dependent materials responses to shock loading are typically based on VISAR measurements alone. Although this approach has proven successful in advancing the materials descriptions, the materials models are ambiguous due to reliance on particle velocity data alone. The particle-velocity-based models are essentially constructed on the volumetric characteristics of the measurements. It has been widely recognized that accurate description of the rate-dependent behaviors require both stress and particle velocity.

The ability to describe both particle velocity and stress with nanosecond time resolution will provide a much more rigorous description for models of rate-dependent materials responses to high pressure shock compression. This capability should remove ambiguity from existing materials models. 


\section{ACKNOWLEDGMENTS}

The authors are pleased to recognize the collaboration with Steve Sheffield and Rick Gustavsen at Los Alamos National Laboratory DX-10, and thank them for their sample preparation and discussions concerning their observations of HMX high explosive behavior. Mel Baer's insight and numerical simulation capabilities have been vital to our progress in developing this new technique. Work supported by the USDOE under contract DE-AC04-94AL85000.

\section{REFERENCES}

1 M. U. Anderson, R. A. Graham, and G. T. Holman, "Time-Resolved Shock Compression of Porous Rutile: Wave Dispersion in Porous Solids", High-Pressure Science and Technology - 1993, eds. S. C. Schmidt, J. W. Shaner, G. A. Samara and M. Ross, AIP Press (1994) pp 1111-1114.

2 R. A. Graham, M. U. Anderson, F. Bauer, and R. E. Setchell, "Piezoelectric Polarization of the Ferroelectric Polymer PVDF from $10 \mathrm{MPa}$ to $10 \mathrm{GPa}$ : Studies of Loading-Path Dependence", Shock Compression of Condensed Matter - 1991, eds. S. C. Schmidt, R. D. Dick, J. W. Forbes, D. G. Tasker, North-Holland (1992) pp 883886.

3 D. E. Wackerbarth, M. U. Anderson, R. A. Graham, SAND92-0046, February, 1992, Sandia National Laboratories.

4 S. A. Sheffield, R. L. Gustavsen, R. R. Alcon, R. A. Graham, and M. U. Anderson, "Particle Velocity and Stress Measurements in Low Density HMX", High-Pressure Science and Technology - 1993, eds. S. C. Schmidt, J. W. Shaner, G. A. Samara, M. Ross, AIP Press (1994) pp 13771380. 